\title{
Midgut Volvulus Due to a True Jejunal Diverticula
}

\author{
Melissa Kyriakos Saad ${ }^{1, \odot}$ Amine Geahchan ${ }^{2}$ Fatimah Ghandour ${ }^{3}$ Fatmeh Ghandour-Hajj ${ }^{3}$ \\ Hani Malouf ${ }^{1} \quad$ Imad El Hajj ${ }^{1} \quad$ Elias Saikaly ${ }^{1, \odot}$
}

${ }^{1}$ Department of General Surgery, Saint George Hospital University

Address for correspondence Elias Saikaly, MD, Saint George

Medical Center, University of Balamand, Beirut, Lebanon

2Department of Radiology, Saint George Hospital University

Medical Center, University of Balamand, Beirut, Lebanon

${ }^{3}$ Department of Pathology, Saint George Hospital University

Medical Center, University of Balamand, Beirut, Lebanon

Hospital University Medical Center, Beirut 961, Lebanon

(e-mail: dreliassaikaly@gmail.com).

Int J Recent Surg Med Sci 2021;7:88-90.
Abstract
Keywords
- jejunal diverticula
- small bowel volvulus
- true diverticulum
Jejunal diverticula is a rare condition quoted to affect between $0.5 \%$ and $7 \%$ of the individuals. This disease is usually clinically silent but becomes symptomatic when complications develop. Among its complications is small bowel volvulus that is a very rare entity.

\section{Introduction}

Small bowel diverticula are sac-like out pouching of the small bowel wall that can occur throughout the small bowel. Small bowel diverticula are most often found in the duodenum, followed by jejunum and ileum. In fact, Akhrass et al found in their retrospective review of 208 patients with symptomatic small bowel diverticulosis that diverticula were located in the duodenum in $79 \%$ of patients, in the jejunum or ileum in $18 \%$, and in all three segments in $3 \% .{ }^{1}$ The incidence of jejunoileal diverticula is variable that is reported in 0.5 to $2.3 \%$ of individuals in radiographic series and up to $7 \%$ in autopsy studies. ${ }^{2}$ Furthermore, $77 \%$ of cases demonstrated multiple diverticula as opposed to solitary diverticula. ${ }^{3}$ They are more commonly reported in men with the highest incidence in the sixth and seventh decades of their life. Small bowel diverticula are usually asymptomatic, and have a spectrum of presentations ranging from nonspecific abdominal pain, dyspepsia, and bloating reaching presentations with life-threatening complications. In fact, Tsiotos et $\mathrm{al}^{4}$ analyzed 112 cases of jejunoileal diverticulosis and of these, $42 \%$ of cases were asymptomatic. In the remaining patients, symptoms of diarrhea were reported in $58 \%$ patients, chronic abdominal pain in $51 \%$, and bloating in $44 \%$. Furthermore, complication rates as high as $46 \%$ for jejunal diverticulosis have been reported and are known to be fatal at sometimes. ${ }^{5}$ Among the very rare complications is small bowel volvulus. Herein, we report a case of small bowel volvulus due to isolated $5 \mathrm{~cm}$ jejunal diverticula in a 55-year-old female patient managed by diverticulectomy and detorsion of the small bowel.

\section{Case Report}

This is a case of a 55-year-old female patient with a previous history of laparoscopic cholecystectomy presenting with 1-day history of nonspecific epigastric pain associated with decreased per os intake, nausea, and two episodes of bilious vomiting. Patient denies fever and change in bowel habits. Patient denies weight loss and personal or family history of malignancy. Upon arrival to our emergency department, her vitals were stable and physical exam of the abdomen was minimal tenderness upon deep palpation of left-sided abdomen. Laboratory workups including complete blood count, inflammatory markers, liver function tests, amylase, lipase, and urine analysis were within normal limits. Computed tomography (CT) scan of the abdomen and pelvis with intravenous contrast was done and showed a whirl sign of the branches of the midportion of superior mesenteric artery with dilatation of the second portion of the duodenum ( - Fig. 1). There was preservation of the normal anatomy of the superior mesenteric vessels as well as the position of the cecum ( - Fig. 2). Furthermore, jejunal loops were noted to published online

November 29, 2021
DOI https://doi.org/

$10.1055 / \mathrm{s}-0040-1721518$ ISSN 2455-7420.
C 2021. Medical and Surgical Update Society.

This is an open access article published by Thieme under the terms of the Creative Commons Attribution-NonDerivative-NonCommercial-License, permitting copying and reproduction so long as the original work is given appropriate credit. Contents may not be used for commercial purposes, or adapted, remixed, transformed or built upon. (https://creativecommons.org/licenses/by-nc-nd/4.0/)

Thieme Medical and Scientific Publishers Pvt. Ltd. A-12, 2nd Floor, Sector 2, Noida-201301 UP, India 


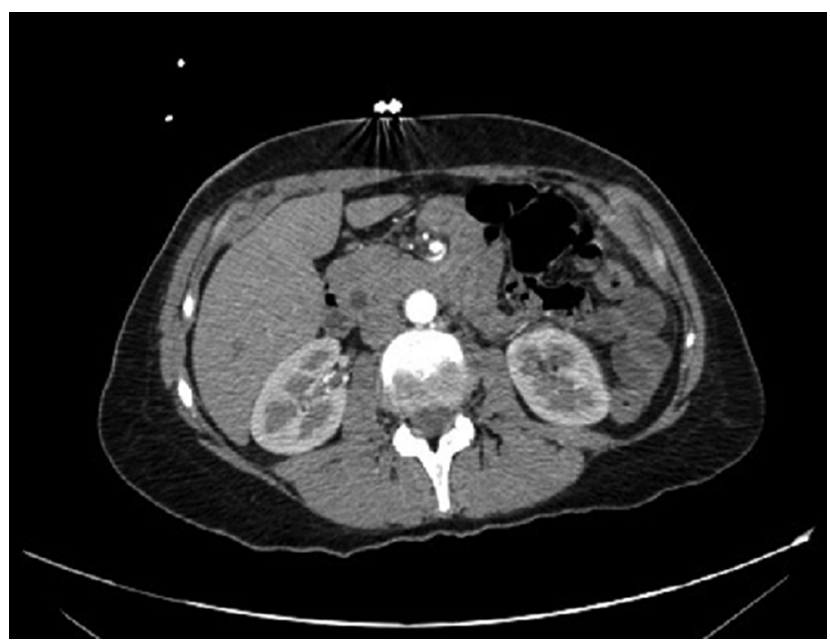

Fig. 1 Arterial phase in this image showing the superior mesenteric artery with its branches swirling pattern with no evidence of distal vascular compromise.

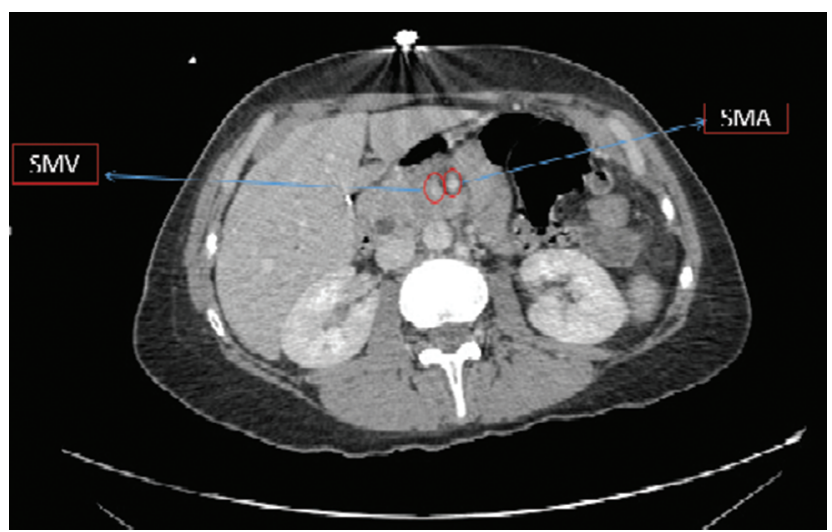

Fig. 2 Portovenous phase in this image showing the superior mesenteric vein (SMV) on the right compared with the superior mesenteric artery (SMA).

be on the right side of the abdomen. At this point, with the diagnosis of midgut volvulus decision was taken for surgical intervention by laparoscopic approach. In the operating theater, a supraumbilical midline open technique was used to insert the $10 \mathrm{~mm}$ trocar, insufflation of the abdomen, and $25 \mathrm{~mm}$ trocars were inserted under direct vision. No compromise of bowel and no anatomical variation were noted; detorsion was done and the cause of volvulus was attributed to the presence of large jejunal diverticula measuring $5 \mathrm{~cm}$ in size. Diverticulectomy was done. Patient had a smooth postoperative stay and was discharged 5 days post-surgery. Histopathologic examination showed the presence of true diverticulum ( - Figs. 3 and $\mathbf{4}$ ), that is, sections of small bowel pouch showed all three layers of bowel wall.

\section{Discussion}

Small bowel diverticulosis was first reported by Sommering in $1794 .{ }^{6}$ Its etiology is thought to be related to a combination between intestinal dyskinesia and abnormal peristalsis causing high segmental intraluminal pressures. ${ }^{7}$ In fact, the current

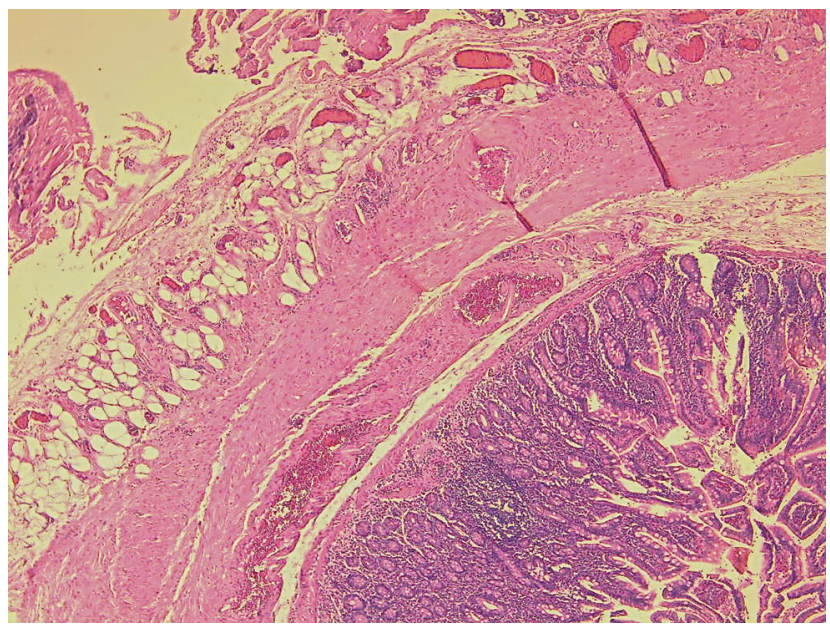

Fig. 3 Intact jejunal mucosa, with normal underlying mucosa and submucosa.

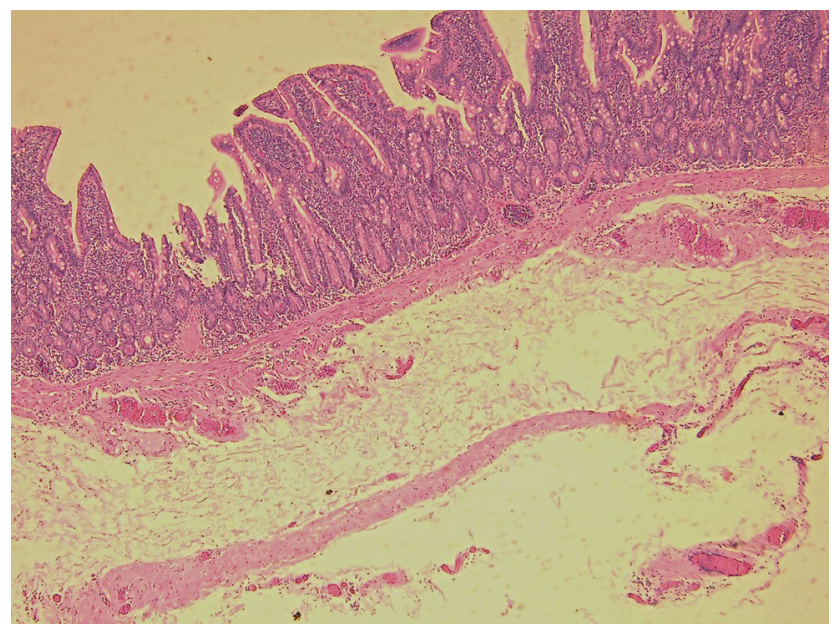

Fig. 4 Focally attenuated muscular layer.

hypothesis focuses on abnormalities in the smooth muscle or myenteric plexus. Having said this, microscopic evaluation of jejunal specimens with diverticulosis has shown that there are three different abnormalities: fibrosis and decreased numbers of normal muscle cells, fibrosis and degenerated smooth muscle cells, and neuronal and axonal degeneration. ${ }^{8}$ The presence of any of the above-mentioned abnormalities will consequently lead to distorted smooth muscle contractions of the affected small bowel generating areas of increased intraluminal pressure, which in turn will lead to herniation of the bowel mucosa and submucosa through the weakest point in the bowel wall, the mesenteric border at the site of entry of the vasa recta, leading to the formation of small bowel diverticula. Small bowel diverticula are usually clinically silent and discovered incidentally or once they become complicated. However, they may have a wide spectrum of presentation ranging from nonspecific abdominal pain, dyspepsia, and bloating reaching presentations with life-threatening complications. Life-threatening complications include massive gastrointestinal bleeding, volvulus, and perforation with localized or generalized peritonitis. Mechanical 
intestinal obstruction occurs in 2.3 to $4.6 \%$ of cases of jejunoileal diverticulosis. ${ }^{9}$ Obstruction can be caused by inflammatory stenosis, intussusceptions, and voluminous jejuna stones. Besides, in jejunoileal diverticulosis, volvulus of the diverticula-containing segment is a cause of intestinal obstruction. Volvulus is a well-established cause of acute small bowel obstruction, more commonly seen in the neonatal period and is extremely rare in adults. Midgut volvulus can be categorized as primary or secondary. Midgut volvulus due to diverticula belongs to the secondary type that could be also caused by postoperative adhesions and tumors. The involved segment, which is filled with fluid and is heavier than the noninvolved part, might be responsible for initiating the volvulus. ${ }^{9}$ Furthermore, it is reported that a large diverticulum was much more frequently seen in patients with volvulus (35\%). ${ }^{10}$ Patients with acute midgut volvulus usually present to the emergency department with the clinical picture of small bowel obstruction. However, those with chronic small bowel volvulus often present atypical symptoms. Examination such as CT angiography aids in the preoperative diagnosis and hence the management. Signs of volvulus on CT scan are whirl signs, intestinal ischemia manifested as thickening of the bowel wall, and free peritoneal fluid and signs of small bowel obstruction. Furthermore, angiographic highlights that aid in the diagnosis of midgut volvulus includes twisted mesenteric vessels, dilated superior mesenteric vein, or prolonged contrast transit time. Proper preoperative management and early surgical treatment are the clue for successful outcome in midgut volvulus. Our case report highlights the importance of maintaining a high clinical suspicion of midgut volvulus in patients presenting with abdomen pain and vomiting as early surgical intervention is the key. Besides, this case highlights the importance of CT scan in detecting midgut volvulus.

\section{Conclusion}

Jejunal diverticulosis remains asymptomatic in $~ 80 \%$ of cases making its diagnosis a challenge to the unaware. Diagnosis is often initiated in cases with related complications, such as hemorrhage, perforation, or obstruction. Intestinal obstruction can be caused by volvulus. Midgut volvulus in adults is a rare entity, even rarer is midgut volvulus due a true jejunal diverticula. A delay in the diagnosis can be fatal because this disease is more common in the elderly population with multiple comorbidities. Early surgical intervention when indicated is the key for successful treatment.

\section{Conflict of Interest}

None declared.

\section{References}

1 Akhrass R, Yaffe MB, Fischer C, Ponsky J, Shuck JM. Small-bowel diverticulosis: perceptions and reality. J Am Coll Surg 1997;184(4):383-388

2 De Peuter B, Box I, Vanheste R, Dymarkowski S. Small-bowel diverticulosis: imaging findings and review of three cases. Gastroenterol Res Pract 2009;2009:549853

3 Lempinen M, Salmela K, Kemppainen E. Jejunal diverticulosis: a potentially dangerous entity. Scand J Gastroenterol 2004;39(9):905-909

4 Tsiotos GG, Farnell MB, Ilstrup DM. Nonmeckelian jejunal or ileal diverticulosis: an analysis of 112 cases. Surgery. 1994;116(4):726--731

5 Davies NM, Saleh JY, Skjodt NM. Detection and prevention of NSAID-induced enteropathy. J Pharm Pharm Sci 2000;3(1):137-155

6 Williams RA, Davidson DD, Serota AI, Wilson SE. Surgical problems of diverticula of the small intestine. Surg Gynecol Obstet 1981;152(5):621-626

7 Kavanagh C, Kaoutzanis C, Spoor K, Friedman PF. Perforated jejunal diverticulum: a rare presentation of acute abdomen. BMJ Case Rep 2014;2014:bcr-2013-202673

8 Krishnamurthy S, Kelly MM, Rohrmann CA, Schuffler MD. Jejunal diverticulosis. A heterogenous disorder caused by a variety of abnormalities of smooth muscle or myenteric plexus. Gastroenterology 1983;85(3):538-547

9 de Bree E, Grammatikakis J, Christodoulakis M, Tsiftsis D. The clinical significance of acquired jejunoileal diverticula. Am J Gastroenterol 1998;93(12):2523-2528

10 Chou CK, Mark CW, Wu RH, Chang JM. Large diverticulum and volvulus of the small bowel in adults. World J Surg 2005;29(1):80-82 